\title{
Depression among Iranian nurses: A systematic review and meta-analysis
}

\author{
Sahar Sadat Saeedi Shahri ${ }^{1}$, Ahmad Ghashghaee ${ }^{1}$, Masoud Behzadifar*2, Nicola Luigi Bragazzi ${ }^{3}$, \\ Meysam Behzadifar ${ }^{4,5}$, Nasrin Mousavinejad ${ }^{5}$, Mozhgan Sadat Ghaemmohamadi ${ }^{1}$, Faezeh Ebadi ${ }^{1}$, Hesam Seyedin ${ }^{6}$
}

\begin{abstract}
Background: Depression is one of the most commonly diagnosed mental disorders. Nurses and other hospital service providers are a group at high risk for developing depression. Thus, knowing the prevalence of depression among nurses can help the health care decisionmakers to plan ad hoc prevention programs to control depression in this group. This study was conducted to quantitatively assess the prevalence of depression in nurses by performing a systematic review and meta-analysis.

Methods: ISI/Web of Science (WoS), PubMed/MEDLINE via Ovid, PsychInfo, and Embase, as well as Iranian databases such as Irandoc, SID, and Magiran were searched from January 2000 to March 2017. To calculate the pooled prevalence rate, the random effects model based on the DerSimonian-Laird approach was used. I2 and the Q tests were used to examine heterogeneity among studies. To investigate the causes and sources of heterogeneity, the impact of such variables as quality, sample size, geographic region, and criteria used to diagnose depression was analyzed performing subgroup analyses. The quality of reviewed studies was assessed according to the 22-item STROBE checklist. Sensitivity analysis was performed to investigate the stability and robustness of the obtained results. All data were analyzed using the "meta" package included in the R Software Version 3.4.0.
\end{abstract}

Results: A total of 30 studies were retained in the current investigation. The overall prevalence of depression was $22 \%$ [ $95 \%$ CI 19 27] among nurses, with a high statistically significant heterogeneity $(I 2=94 \%$ and Q-test $=479.87)$.

Conclusion: This rate was higher than the prevalence of depression among the general population in Iran. Presence of happy nurses with good mental state at hospitals is essential for promoting the care provided to patients.

Keywords: Depression, Nurses, Iran, Systematic Review, Meta-analysis

Copyright $\odot$ Iran University of Medical Sciences

Cite this article as: Saeedi Shahri SS, Ghashghaee A, Behzadifar M, Luigi Bragazzi N, Behzadifar M, Mousavinejad N, Sadat Ghaemmohamadi M, Ebadi F, Seyedin H. Depression among Iranian nurses: A systematic review and meta-analysis. Med J Islam Repub Iran. 2017 (25 Dec);31:130. https://doi.org/10.14196/mjiri.31.130

\section{Introduction}

Depression is one of the most commonly diagnosed mental disorders. According to the World Health Organization (WHO), about 300 million people suffer from depression worldwide, which represents one of the leading causes of disability and global burden of diseases (1). Every year, large amounts of money are spent to treat depressed patients (2). This disorder imposes a considerable economic

Corresponding author: Masoud Behzadifar, masoudbehzadifar@gmail.com

1. Student Research Committee, School of Health Management and Information Sciences Branch, Iran University of Medical Sciences, Tehran, Iran.

2. Health Management and Economics Research Center, Iran University of Medical Sciences, Tehran, Iran.

3. School of Public Health, Department of Health Sciences (DISSAL), University of Genoa, Genoa, Italy.

${ }^{4}$ Social Determinants of Health Research Center, Lorestan University of Medical Sciences, Khorramabad, Iran.

5. Department of Epidemiology, Faculty of Health \& Nutrition, Lorestan University of Medical Sciences, Khorramabad, Iran.

${ }^{6}$. Department of Health Services Management, School of Health Management and Information Sciences, Iran University of Medical Sciences, Tehran, Iran. burden on the society; therefore, prevention plays an important role in saving resources and improving quality of life (3).

By 2030, depression is expected to become the second cause of disability and comorbidities in developing countries (4), profoundly impacting people's performance and quality of life. Furthermore, an anticipation of age-of-onset

$\uparrow$ What is "already known" in this topic:

Depression is one of the most commonly diagnosed mental disorders. Physicians, nurses, and other hospital service providers represent a group of workers at high risk for developing depression.

\section{$\rightarrow$ What this article adds:}

The overall prevalence of depression among nurses is $22 \%$, and this rate is higher than the prevalence of depression among the general population in Iran. Thus, knowing the prevalence of depression among nurses can help the health care decision-makers to plan ad hoc prevention programs to control depression in this working group. 
is expected (5). From an etiopathogenetic standpoint, hereditary genetic and biochemical causes can cause depression, disturbing communication between nerve cells (6). Social and family problems and employment conditions can be among the causes of depression (7). This disease severely impairs familiar and social sphere, as well as professional working, leading, in some cases, to suicide (8).

Doctors, nurses, and other hospital service providers are among a group at high risk for developing depression (9). It is estimated that depression has a greater impact on job performance than chronic diseases, such as arthritis, blood pressure, backache, and diabetes (10). Nursing is among occupational groups at high risk for depression due to harsh working conditions (11).

Several studies have examined the prevalence of depression among nurses. In 2 studies conducted in the USA, the prevalence of depression was reported to range from $18 \%$ to $41 \%(12,13)$. In a study conducted in China, this rate was about $38 \%$ (14). In Iran, various studies have investigated the prevalence of depression among nurses. Thus, knowing the prevalence of depression among nurses can help the health care decision- makers to plan ad hoc prevention programs to control depression in this working group.

When nurses have good physical and mental status, they are likely to make less job mistakes and their working motivation increases. The use of psychiatric services for nurses, who are prone to depression, should be considered.

Therefore, this study was conducted to quantitatively assess the prevalence of depression in nurses by systematic review and meta-analysis method in Iran.

\section{Methods}

\section{Literature search}

The results of the current investigation were reported according to the "Preferred Reporting Items for Systematic Reviews and Meta-Analyses" (PRISMA) guidelines. Several databases including ISI/Web of Science (WoS), PubMed/MEDLINE via Ovid, PsychInfo, and Embase, as well as Iranian databases such as Irandoc, SID, and Magiran were searched from January 2000 until March 2017. The search strategy was as follows: "Prevalence" AND ("Depression" OR "Mental Health Disorder" OR "Major Depression Disorder") AND "Nurses" AND "Iran". Search strategy is reported in Appendix 1. The reference lists of potentially eligible articles were scanned to find further relevant studies. Two authors independently eliminated duplicate studies, screened them based on title and abstract, and finally, identified studies to be retained based on inclusion/exclusion criteria.

\section{Inclusion and exclusion criteria}

We included studies if they were:

1. Observational studies (cross-sectional, case-control, cohort investigations) examining the prevalence of depression among Iranian nurses with clear and unambiguous results;

2. Studies published in both English and Persian;

3. Studies published between January 2000 and March 2017;
4. Studies using validated/standardized tools for examining depression.

We excluded studies if they were:

1. Studies designed as randomized clinical trials (RCTs), case-report investigations, case series, reviews, and editorials;

2. Studies reporting overlapping results;

3. Studies whose results were not clear, and in which the possibility of calculating the prevalence was not provided.

\section{Data extraction and quality assessment of articles}

Two of the authors extracted the required data after agreeing on selected studies. Disagreements were resolved by discussion. Finally, the name of the first author, year of publication, number of participants, age, city of the study, province of the study, type of test used to assess depression, and the prevalence of reported depression were extracted and collected in ad hoc designed form.

The quality of reviewed studies was assessed according to the 22-item "Strengthening the Reporting of Observational Studies in Epidemiology" (STROBE) checklist (15). This assessment was performed independently by 2 authors, and their eventual disagreements were resolved by discussion.

\section{Statistical analysis}

To calculate the pooled prevalence rate, the random-effects model based on the DerSimonian-Laird approach was used. Results were computed with their $95 \%$ confidence interval (CI). P-value $<0.05$ was considered as statistically significant. The $\mathrm{I}^{2}$ and the $\mathrm{Q}$ tests were used to examine heterogeneity among studies (16). To investigate the causes and sources of heterogeneity, the impact of such variables as quality, sample size, geographic region, and criteria used to diagnose depression was analyzed performing subgroup analyses. Sensitivity analysis was performed to investigate the stability and robustness of the obtained results. In addition, cumulative meta-analysis was done based on year of publication. To explore more sources of heterogeneity, meta-regression was used based on the publishing year and sample, according to the previously defined variables. Egger's test was used to analyze publication bias (17). All data were analyzed using the "meta" package included in the R Software Version 3.4.0 (freely available and downloadable at https://www.r-project.org/).

\section{Results}

After the initial search and selecting pertinent studies based on inclusion/exclusion criteria, 30 studies were finally included (18-47). Figure 1 displays the process of finding and selecting relevant studies. The overall number of included participants was 5613. The design of all included studies was cross-sectional.

Sample sizes ranged from 60 to 413 . The main characteristics of the included studies are presented in Table 1 . The overall prevalence of depression among nurses was $22 \%$ [95\%CI 19-27], with $\mathrm{I}^{2}=94 \%$ and Q-test $=479.87$ (Fig. 2).

The results of the subgroup analyses based on quality, sample size, geographic region, and diagnostic test used are demonstrated in Table 2. 


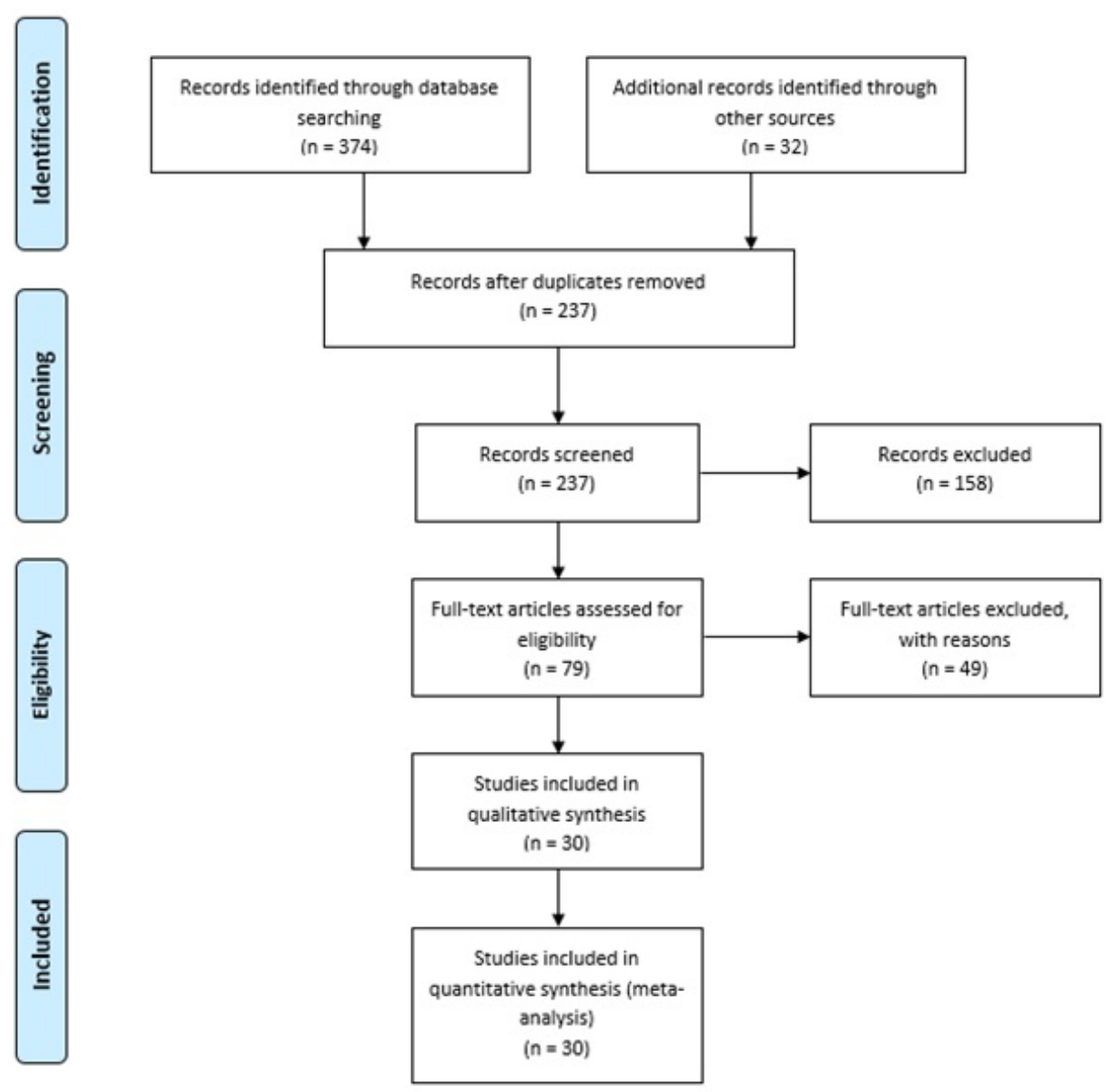

Fig. 1. Flowchart of the current systematic review and meta-analysis

\begin{tabular}{|c|c|c|c|c|c|c|}
\hline First author & Year & Sample & Questionnaire & Quality & City & Age \\
\hline Khajeh nasiri & 2000 & 130 & Beck & High & Tehran & NA \\
\hline Khalil zadeh & 2005 & 200 & Beck & High & Urmia & NA \\
\hline Habibzadeh & 2005 & 270 & Goldberg & Medium & Khoy & NA \\
\hline Abedini & 2007 & 95 & Beck & High & Bandar abbas & $21.13 \pm 2.03$ \\
\hline Arefian & 2009 & 400 & Beck & High & Tehran & NA \\
\hline Karami & 2009 & 208 & Beck & High & Kashan & $20-24$ \\
\hline Dehghani & 2009 & 311 & Zung Self-Rating Depression Scale & Medium & Shiraz & $31.24 \pm 8.21$ \\
\hline Mirmohammadi & 2009 & 110 & Beck & High & Yazd & $38.46 \pm 5.48$ \\
\hline Asadzandi & 2011 & 272 & Dass & High & Tehran & $38.69 \pm 7.8$ \\
\hline Khamseh & 2011 & 413 & Dass & Medium & Tehran & NA \\
\hline Baghiani moghadam & 2012 & 125 & Beck & High & Yazd & $22 \pm 3.2$ \\
\hline Ghasemnegad & 2012 & 150 & Beck & High & Lahijan & $19-27$ \\
\hline Masoudi asl & 2012 & 200 & Beck & High & Yasouj & NA \\
\hline Hadavi & 2012 & 64 & Beck & High & Rafsanjan & $20.10 \pm 1.35$ \\
\hline Pournamdarian & 2012 & 120 & Dass & Low & Hamedan & NA \\
\hline Mahmodi & 2013 & 314 & Beck & High & Sanandaj & NA \\
\hline Nazemi & 2013 & 244 & GDS & Low & Tehran & NA \\
\hline Mami & 2014 & 117 & Beck & High & Ilam & NA \\
\hline Kassani & 2014 & 191 & Beck & High & Ilam & NA \\
\hline Taghva & 2014 & 173 & Beck & High & Tehran & $45 \pm 6.63$ \\
\hline Taghinejad & 2014 & 81 & GHQ & Medium & Ilam & NA \\
\hline Tajvar & 2015 & 72 & GHQ & Medium & Bandar abbas & 31.57 \\
\hline
\end{tabular}

\section{Sensitivity Analysis}

Sensitivity analysis revealed no change in the results, and thus, ensured the stability of the findings (Appendix 2).

\section{Cumulative analysis}

Studies were sorted based on year of publication, and no statistically significant change could be noticed in the results (Appendix 3). 
Meta-analysis of depression in Iranian nurses

\begin{tabular}{|c|c|c|c|c|c|c|}
\hline \multicolumn{7}{|l|}{ Table 1. Cntd } \\
\hline Alipoor & 2015 & 142 & Dass & Low & Fasa & $35 \pm 8.6$ \\
\hline Yasemi & 2015 & 60 & Dass & Low & Ilam & $31.86 \pm 8.35$ \\
\hline Bahnam & 2016 & 182 & Beck & High & Semnan & $29.74 \pm 7.919$ \\
\hline Khani & 2016 & 196 & Beck & High & Neishabour & $32.1 \pm 8$ \\
\hline Yazdanshenas & 2016 & 94 & Dass & Low & Tehran & NA \\
\hline Khodadadi & 2016 & 242 & Dass & High & Tabriz & NA \\
\hline Kashani & 2017 & 102 & MMPI & Medium & Tehran & $30.2 \pm 5.6$ \\
\hline Sagharjoghi Farahani & 2017 & 241 & Beck & High & Khoram abad & NA \\
\hline
\end{tabular}

\section{Study}

Khajeh nasiri 2000

Khalil zadeh 2005

Habibzadeh 2005

Abedini 2007

Arefian 2009

Karami 2009

Dehghani 2009

Mirmohammadi 2009

Asadzandi 2011

Khamseh 2011

Baghiani moghadam 2012

Ghasemnegad 2012

Masoudi asl 2012

Hadavi 2012

Pournamdarian 2012

Mahmodi 2013

Nazemi 2013

Mami 2014

Kassani 2014

Taghva 2014

Taghinejad 2014

Tajvar 2015

Alipoor 2015

Yasemi 2015

Bahnam 2016

Khani 2016

Yazdanshenas 2016

Khodadadi 2016

Kashani 2017

Sagharjoghi Farahani 2017

Random effects model

Heterogeneity: $I^{2}=94 \%, \tau^{2}=0.2229, p<0.01$

Events Total

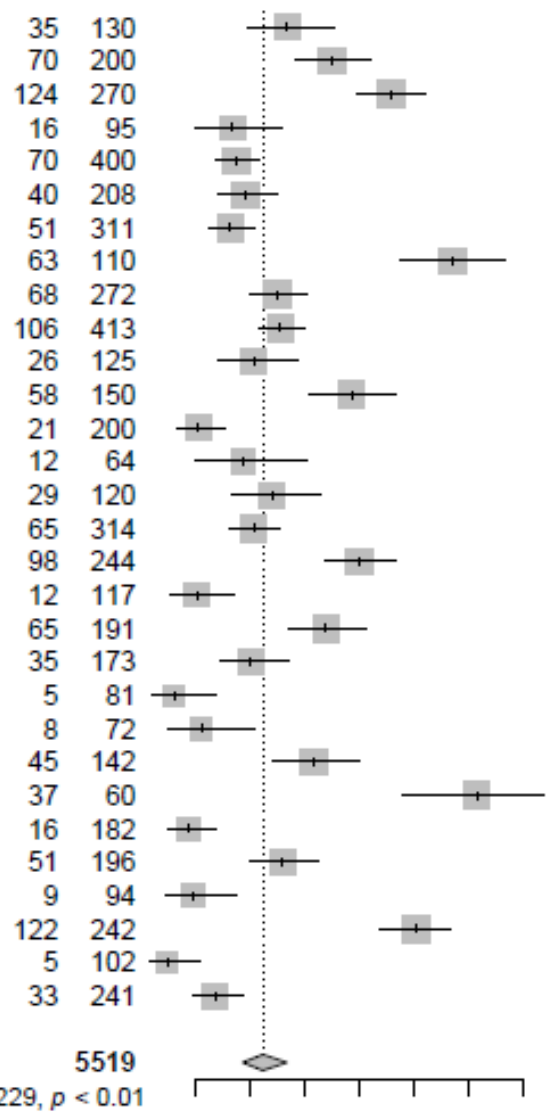

$\begin{array}{lllllll}0.1 & 0.2 & 0.3 & 0.4 & 0.5 & 0.6 & 0.7\end{array}$

\section{Proportion $\quad 95 \%-\mathrm{Cl}$ Weight}

$0.27[0.20 ; 0.35] \quad 3.5 \%$

$0.35[0.28 ; 0.42] \quad 3.7 \%$

$0.46[0.40 ; 0.52] \quad 3.7 \%$

$0.17[0.10 ; 0.26] \quad 3.1 \%$

$0.18[0.14 ; 0.22] \quad 3.6 \%$

$0.19[0.14 ; 0.25] \quad 3.5 \%$

$0.16[0.12 ; 0.21] \quad 3.6 \%$

$0.57[0.47 ; 0.67] \quad 3.7 \%$

$0.25[0.20 ; 0.31] \quad 3.6 \%$

$0.26[0.22 ; 0.30] \quad 3.7 \%$

$0.21[0.14 ; 0.29] \quad 3.4 \%$

$0.39[0.31 ; 0.47] \quad 3.6 \%$

$0.10[0.07 ; 0.16] \quad 3.2 \%$

$0.19[0.10 ; 0.30] \quad 2.9 \%$

$0.24[0.17 ; 0.33] \quad 3.4 \%$

$0.21[0.16 ; 0.26] \quad 3.6 \%$

$0.40[0.34 ; 0.47] \quad 3.7 \%$

$0.10[0.05 ; 0.17] \quad 2.9 \%$

$0.34[0.27 ; 0.41] \quad 3.7 \%$

$0.20[0.15 ; 0.27] \quad 3.5 \%$

$0.06[0.02 ; 0.14] 2.1 \%$

$0.11[0.05 ; 0.21] \quad 2.5 \%$

$0.32[0.24 ; 0.40] \quad 3.6 \%$

$0.62[0.48 ; 0.74] \quad 3.7 \%$

$0.09[0.05 ; 0.14] \quad 3.0 \%$

$0.26[0.20 ; 0.33] \quad 3.6 \%$

$0.10[0.04 ; 0.17] \quad 2.6 \%$

$0.50[0.44 ; 0.57] \quad 3.8 \%$

$0.05[0.02 ; 0.11] \quad 2.1 \%$

$0.14[0.10 ; 0.19] \quad 3.4 \%$

$0.22[0.19 ; 0.27] 100.0 \%$

Fig. 2. Forest plot of the included studies

Table 2. Subgroup analyses of the included studies

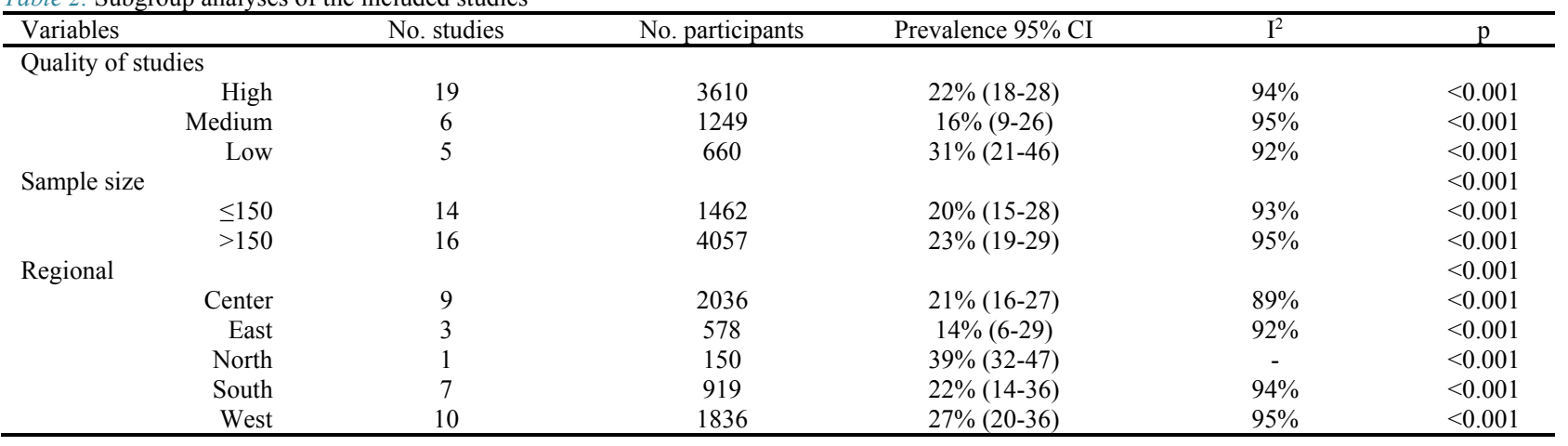

The results of sensitivity analysis and cumulative analysis showed that the overall prevalence did not change in a statistically significant way.
Meta- regression

Meta-regression was conducted based on year of publication and sample size. Results showed that the prevalence of depression in Iranian nurses tended to decrease based on the publishing year, and this decrease was statistically significant. 


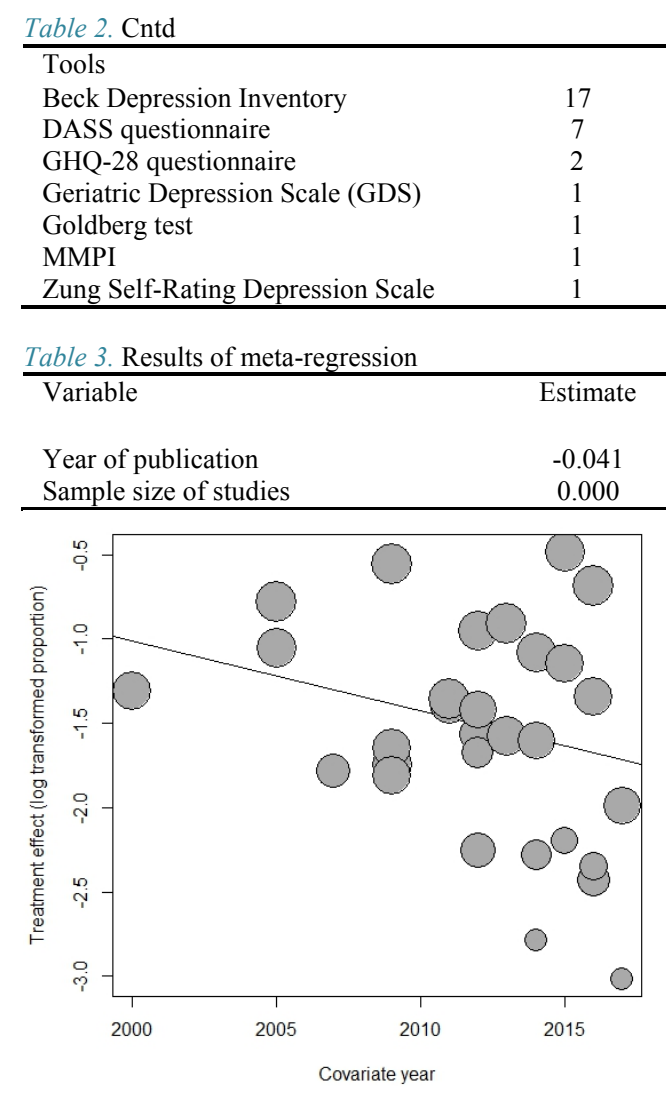

Fig. 3. Results of the meta-regression analyses

Meta-regression based on the sample size of the included studies showed that depression prevalence tended to increase, but this increase was not statistically significant (Table 3 and Fig. 3).

\section{Publication bias}

Publication bias was assessed with Egger's test. P-value was 0.412 , showing no significant evidence of publication bias.

\section{Discussion}

Studies examining the prevalence of diseases and health problems can provide useful and valuable information for health decision-makers, helping them to properly implement interventional programs and secondary and tertiary level prevention activities (48). In this study, the prevalence of depression was studied among the Iranian nurses. Based on these results, prevalence of depression was $22 \%$. The prevalence among Iranian general population was reported to be $4 \%$. The results of this study showed that depression among nurses is significantly higher than the overall pooled prevalence rate in Iran (49).

In a study conducted in China, the prevalence of depression among nurses was 38\% (14). Also in 2 studies conducted in Taiwan, the prevalence was $52.5 \%$ and $27.7 \%$, respectively $(50,51)$. Canada, France, and the USA reported a prevalence rate of $10 \%$ to $40 \%(12,13,52,53)$.

Various factors, such as differences in personality, cultural, social, and working conditions may explain differ- ences in the prevalence of depression among nurses in different countries around the world. Moreover, different methodological designs and different tools used for assessing depression could play a role in explaining these discrepancies.

The rate of depression among nurses is considerably high. Nursing is considered as one of the most stressful jobs, and this can increase depression and reduce nurses' job performance and working motivation over time (54).

In Iran, the Ministry of Health is responsible for recruiting and training nurses. Given the limited financial resources in the health sector, the country is faced with shortage of nurses like other many developing countries. A total of 90026 nurses in Iran were employed in 2008, while the country needed at least approximately 220000 nurses (55). The shortage of work force has led the nurses to provide services for longer hours. Lack of manpower in hospitals increases job burnout in nurses, reduces job satisfaction, and raises the rate of depression (56). Based on studies in different geographical areas of Iran and considering the results of this study, the highest rate of depression was in the Western part of Iran, with a prevalence of $27 \%$. Iran has a large population in its West part, and unfortunately, shortage of nurses is particularly high in this area. On the other hand, the significant role of the private sector in the health sector and the lack of private hospitals have increased the workload of nurses, resulting in an increase in the rate of depression among them.

Several studies have shown that nurses who work for longer periods are more prone to depression. Fatigue 
caused by an increase in working hours can have a devastating impact on intellectual and spiritual life (52). Also, the rate of depression among nurses could depend on working in specific wards, where stress is higher. Unfortunately, in this study, no proper information was available to compare the level of depression in different parts of hospitals.

It seems that health care workers working in intensive care units (ICUs), coronary care units (CCUs), emergency, surgery, pediatrics, ear, nose and throat (ENT), and internal departments are at higher risk of developing depression. Also, nurses working in psychiatric hospitals and clinics are more prone to depression. Due to hard working conditions in these sectors, the nurses are at greater risk for developing depression $(52,57)$. On the other hand, in sectors such as infectious diseases, fear of infections may cause too much stress in nurses. Nurses' work stress is generally high, and as the most important reason for depression is stress, stress management plays an important role in reducing depression in nurses (57). On the other hand, violence in the workplace is another factor profoundly influencing nurses' mental health. The violent and intimidating behavior of some patients and their relatives is another reason for the increase in depression rate (58). Studies revealed a relationship between workplace violence and depression among nurses. Indeed, nurses who have experienced violence are more likely to develop depression than the ones who have been less exposed to violence. Research shows that the prevalence of violence caused by employment condition has a huge impact on psychological conditions of nurses (59).

Nurses, due to long working hours in the hospital, do not have a chance to attend social activities. Sustained engagement in social activities, communication with friends, and emotional interactions with family members make people less susceptible to stress, isolation, and depression (60).

Research has shown that people who work in rotating shifts (round the clock) have lower level of serotonin hormone than others. Serotonin is a neurotransmitter of the central nervous system that helps the body to regulate sleep. Insomnia has a negative impact on the body and mind of individuals over time and can cause depression and anxiety. Working shifts can cause insomnia and disruption of the body's circadian rhythm. Nurses and doctors are among people whose circadian rhythm is disrupted and get insufficient sleep. This impairs family, work, and social spheres $(61,62)$.

In this study, the prevalence of depression ranged from $5 \%$ to $46 \%$ (Table 2 ). One of the reasons for this variation comes from differences in diagnostic tests used (63).

The strengths of this study were performing a comprehensive and systematic research on diverse and important medical sites, using sensitivity analysis to check the stability of the results, and studying the potential sources of heterogeneity by meta-regression and subgroups-analyses.

However, this study had some limitations. There was a high level of observed heterogeneity, which was mainly due to the examination of studies characterized by different geographic locations, and there were different methodological features. Furthermore, the collected data were insufficient to stratify the prevalence of depression by gender and different hospital wards.
As such, we recommend that future high-quality studies be conducted fully taking into account the results of the current investigation.

\section{Conclusion}

According to the results of this study, the prevalence of depression among Iranian nurses is $22 \%$, which is relatively high. Presence of happy nurses with good mental state is essential for the promotion of patients' health care. Thus, health policy- makers in Iran should have a comprehensive plan to increase the manpower in nursing, try to manage nurses' working hours, and provide wage increase and welfare services to raise job satisfaction and motivation in nurses.

\section{Funding/Support}

Iran University of Medical Sciences, Tehran, Iran, (Grant No: 96-01-193-30016).

\section{Conflict of Interests}

The authors declare that they have no competing interests.

\section{References}

1. World health organization. Depression. 2017; Available from: http://www.who.int/mediacentre/factsheets/fs369/en/.

2. Okumura Y, Higuchi T. Cost of depression among adults in Japan. Prim Care Companion CNS Disord. 2011;3:pii: PCC.10m01082.

3. Luppa M, Heinrich S, Angermeyer MC, König HH, Riedel-Heller SG. Cost-of-illness studies of depression: a systematic review. J Affect Disord. 2007;98:29-43

4. Mathers CD, Loncar D. Projections of global mortality and burden of disease from 2002 to 2030. PLoS Med. 2006;3:e442.

5. Ustün TB, Ayuso-Mateos JL, Chatterji S, Mathers C, Murray CJ. Global burden of depressive disorders in the year 2000. Br J Psychiatry. 2004;184:386-92.

6. Goodwin GM. Depression and associated physical diseases and symptoms. Dialogues Clin Neurosci. 2006;8:259-65.

7. Kessler RC, Greenberg PE, Mickelson KD, Meneades LM, Wang PS The effects of chronic medical conditions on work loss and work cutback. J Occup Environ Med. 2001;43:218-25.

8. Mata DA, Ramos MA, Bansal N, Khan R, Guille C, Di Angelantonio E, et al. Prevalence of Depression and Depressive Symptoms Among Resident Physicians: A Systematic Review and Meta-analysis. JAMA. 2015;314:2373-83.

9. Callaghan P, Tak-Ying SA, Wyatt PA. Factors related to stress and coping among Chinese nurses in Hong Kong. J Adv Nurs. 2000;31: 1518-27.

10. Kessler RC, EJ B. The epidemiology of depression across cultures. Annu Rev Public Health. 2013;34:119-38.

11. Oates J, Drey N, Jones J. Associations between Age, Years in Post, Years in the Profession and Personal Experience of Mental Health Problems in UK Mental Health Nurses. Issues Ment Health Nurs. 2017:1-9

12. Letvak S, Ruhm CJ, McCoy T. Depression in hospital-employed nurses. Clin Nurse Spec. 2012;26:177-82.

13. Ruggiero JS. Health, work variables, and job satisfaction among nurses. J Nurs Adm. 2005;35:254-63.

14. Gong Y, Han T, Yin X, Yang G, Zhuang R, Chen Y, et al. Prevalence of depressive symptoms and work-related risk factors among nurses in public hospitals in southern China: A cross-sectional study. Sci Rep. 2014;4:7109.

15. Vandenbroucke JP, von Elm E, Altman DG, Gøtzsche PC, Mulrow $\mathrm{CD}$, Pocock SJ, et al. Strengthening the Reporting of Observational Studies in Epidemiology (STROBE): explanation and elaboration. Int J Surg. 2014;12:1500-24.

16. Higgins JP, Thompson SG, Deeks JJ, Altman DG. Measuring inconsistency in meta-analyses. BMJ. 2003;327:557-60.

17. Egger M, Davey Smith G, Schneider M, Minder C. Bias in meta- 
analysis detected by a simple, graphical test. BMJ. 1997;315:629-34.

18. Abedini S, Davachi A, Sohbaee F, Mahmoodi M, Safa O. Prevalence of depression in nursing students in Hormozgan University of Medical Sciences. HMJ. 2007;11:2.

19. Alipoor R, Ebrahimi A, Omidi R, Hedayati A, Ranjbar H, Hosseinpour S. Depression, anxiety, stress and related demographic variables in nurses of Valiasr hospital in Fasa University of Medical Sciences in 2014. Pajouhan Sci J. 2015;13:51-9.

20. Arefian NM, Seddighi A, Seddighi AS, Nobahar MR. Depression in the nurses of the special wards versus nurses of the general wards, a comparative study. Iran J Cancer Prev. 2009;2:151-4.

21. Asad Zandi M, Sayari R, Ebadi A, Sanainasab H. Abundance of depression, anxiety and stress in militant Nurses. J Mil Med. 2011; 13:103-8.

22. Baghiani Moghaddam MH, Ehrampoush MH, Rahimi B, Aminian AH, Aram M. Prevalence of depression among successful and unsuccessful students of Public Health and Nursing-Midwifery schools of Shahid Sadoughi University of Medical Sciences in 2008. JMED. 2012;6:17-24.

23. Bahnam B, Semnani V, Hadadnia F, Mirmohammadkhani M. Vitamin D serum levels in nurses in Semnan educational hospitals and its association with depression. Koomesh J. 2016;17:313-22.

24. Dehghani M, Zoladl M, Boland-Parvaz SH, Keshtkaran Z, Mahmoudi R, Jabbarnejad A. A survey on depression and its related factors in Nurses who work in Namazi Hospital of Shiraz University of Medical Sciences-2008. Iran Occup Health. 2009;6:29-34.

25. Ghasemnegad SM, Barchordary M. Frequency of anxiety and its relation with depression and other individual characteristics in nursing students. J Holist Nurs Midwifery. 2012;22.

26. Habibzadeh H, Motearefi H, Hassanpoor dehkordi A. Risk factors of depression among nursing students in West Azarbaijan province. Mod Care J. 2005;2:23-7.

27. Hadavi M, Rostami N. Depression and its effective factors among the students of Rafsanjan Nursing, Midwifery and Paramedical faculty- 2012. Community Health J. 2012;6:58-65.

28. Karami M. The study of the rate of depression in allied health faculty students of kashan university of medical sciences in 2008. J Urmia Nurs Midwifery Fac. 2009;7.

29. Kashani P, Mirbaha S, Forouzanfar MM, Meschi F, Baratloo A. The Prevalence of Personality Disorders among Emergency Nurses Based on MMPI-2 Questionnaire; a Cross-sectional Study. Emergency. 2017;5.

30. Kassani A, Niazi M, Menati R, Alimohamadi Y, Menati W. Relationship between nurses' depression and quality of life: applying path analysis model. Q J Nurs Manag. 2014;3:61-9.

31. Khajeh Nasiri F. A study of depression prevalence of nurses and its effective factors in Tehran Emam Khomeini Hospital. Tehran Univ Med J. 2000;58:10-4.

32. Khalilzadeh R, Yavarian R, Khalkhali R. The relationship of job stress, depression and anxiety of nursing staff of Urmia University of Medical Sciences. J Urmia Nurs Midwifery Fac. 2005;3.

33. Khamseh F, Roohi H, Ebaady A, Hajiamini Z, Salimi H, Radfar S. Survey Relationship between demographic factors and stress, anxiety and depression in nurses working in selected hospitals in Tehran city . J Holist Nurs Midwifery. 2011;21:13-21.

34. Khani H, Ghodsi H, Nezhadnik H, Teymori S, Ghodsi A. Depression and its relationship with hypochondriasis in nurses in Neyshabur, Iran. Mil Caring Sci. 2016;3:34-40.

35. Khodadadi E, Hosseinzadeh M, Azimzadeh R, Fooladi M. The relation of depression, anxiety and stress with personal characteristics of nurses in hospitals of Tabriz, Iran. Int J Med Res Health Sci. 2016;5:140-8.

36. Mahmodi SH, Zehni K. The comparison of depression prevalence between shift work nurses in education hospitals of kurdistan medical sciences university. Iran J Nurs Res. 2013;8:29-38.

37. Mami S, Mehdian K, Davoodian Z. Investigation of depression rate in nurses working at state hospitals of the city of and its associated factors. SJIMU. 2014;22:51-6.

38. Masoudi Asl I. Evaluation of Depression in Nursing Students of Azad University. Middle East J Disabil Stud. 2012;1:42-6.

39. Mirmohammadi S, Mehrparvar A, FallahMehrjerdi A. The effect of shift work on depression in nurses. TKJ. 2009;1:24-7.

40. Nazemi L, SKOOG I, Karlsson I, Hosseini S, Hosseini M, Hosseinzadeh MJ, et al. Depression, prevalence and some risk factors in elderly nursing homes in Tehran, Iran. Iran J Public Health.
2013;42:559

41. Purnamdarian S, Birashk B, Asghar nejad $\mathrm{f}$. The Clarification of Contribution of Meta-Cognitive BelieFs in Explaining the Symptoms of Depression, Anxiety and Stress in Nurses. Knowl Res Appl Psychol. 2012;13:86-94.

42. Sagharjoghi Farahani M, Khodadadi B, Dalvand S. The Study of the Prevalence of Depression and Associated Factors among Nurses in Khoram Abad Hospitals during 2016. J Nurs Midwifery Khorramabad. 2017;10.

43. Taghinejad H, Suhrabi Z, Kikhavani S, Jaafarpour M, Azadi A. Occupational mental health: A study of work-related mental health among clinical nurses. J clinic diagn Res, 2014;8.

44. Taghva A, Yazdani A, Ebrahimi MR, Alizadeh K, Sakhabakhsh M. Prevalence of depression in psychiatric nurses and comparison with other parts of the AJA hospitals. NPWJM. 2014;1:11-6.

45. Tajvar A, Saraji GN, Ghanbarnejad A, Omidi L, Hosseini SSS, Abadi ASS. Occupational stress and mental health among nurses in a medical intensive care unit of a general hospital in Bandar Abbas in 2013. Electron Physician. 2015;7:1108.

46. Yasemi M, Peyman H, Khajavikhan J, Nasiri AA, Najafi F, Hemati K, Bimanand Lida. Prevalence of Depression among Nurses Working in the Operating Rooms and Intensive Care Units. J zabol Uni Med Sci Health Serv. 2015;6:70-7.

47. Yazdanshenas Ghazwin M, Kavian M, Ahmadloo M, Jarchi A, Javadi SG, Latifi S, Tavakoli SAH, Ghajarzadeh M. The Association between Life Satisfaction and the Extent of Depression, Anxiety and Stress among Iranian Nurses: A Multicenter Survey. Iran J Psychiatr. 2016;11:120

48. Abubakar I, Aliyu SH, Arumugam C, Usman NK, Hunter PR. Treatment of cryptosporidiosis in immunocompromised individuals: systematic review and meta-analysis. Br J Clin Pharmacol. 2007;63: $387-93$

49. Sadeghirad B, Haghdoost AA, Amin-Esmaeili M, Shahsavand Ananloo E, Ghaeli P, Rahimi-Movaghar A, et al. Epidemiology of Major Depressive Disorder in Iran: a Systematic Review and MetaAnalysis. Int J Prev Med. 2010;1:81-91.

50. Chang Y, Wang PC, Li HH, Liu YC. Relations among depression, self-efficacy and optimism in a sample of nurses in Taiwan. J Nurs Manag. 2011;19:769-76.

51. Lin HS, Probst JC, Hsu YC. Depression among female psychiatric nurses in southern Taiwan: main and moderating effects of job stress, coping behaviour and social support. J Clin Nurs. 2010;19: 2342-54.

52. Nourry N, Luc A, Lefebvre F, Sultan-Taïeb H, Béjean S. Psychosocial and organizational work environment of nurse managers and self-reported depressive symptoms: cross-sectional analysis from a cohort of nurse managers. Int J Occup Med Environ Health. 2014;27:252-69.

53. Ohler MC, Kerr MS, Forbes DA. Depression in nurses. Can J Nurs Res. 2010;42:66-82.

54. Tsai YC, Liu CH. Factors and symptoms associated with work stress and health-promoting lifestyles among hospital staff: a pilot study in Taiwan. BMC Health Serv Res. 2012;16:199.

55. Zarea K, Negarandeh R, Dehghan-Nayeri N, Rezaei-Adaryani M. Nursing staff shortages and job satisfaction in Iran: issues and challenges. Nurs Health Sci. 2009;11:326-31.

56. Zhang LF, You LM, Liu K, Zheng J, Fang JB, Lu MM, et al. The association of Chinese hospital work environment with nurse burnout, job satisfaction, and intention to leave. Nurs Outlook. 2014; 62:128 37.

57. Arimura M, Imai M, Okawa M, Fujimura T, Yamada N. Sleep, mental health status, and medical errors among hospital nurses in Japan. Ind Health. 2010;48:811-7.

58. Rafati Rahimzadeh M, Zabihi A, Hosseini S. Verbal and Physical Violence on Nurses in hospitals of Babol University of Medical Sciences. Hayat. 2011;17:5-11.

59. Merecz D, Drabek M, Mościcka A. Aggression at the workplace-psychological consequences of abusive encounter with coworkers and clients. Int J Occup Med Environ Health. 2009;22:243-60.

60. Diener E, Oishi S, Lucas RE. Personality, culture, and subjective well-being: emotional and cognitive evaluations of life. Annu Rev Psychol. 2003;54:403-25.

61. Bannai A, Tamakoshi A. The association between long working hours and health: a systematic review of epidemiological evidence. Scand J Work Environ Health. 2014;40:5-18.

62. Chiou ST, Chiang JH, Huang N, Wu CH, Chien LY. Health issues 
among nurses in Taiwanese hospitals: National survey. Int J Nurs Stud. 2013;50:1377-84

63. Ravaghi H, Behzadifar M, Behzadifar M, Taheri Mirghaed M, Aryankhesal A, Salemi M, et al. Prevalence of Depression in
Heaaadxxc ,modialysis Patients in Iran: a Systematic Review and Meta-analysis. Iran J Kidney Dis. 2017;11:90-8.

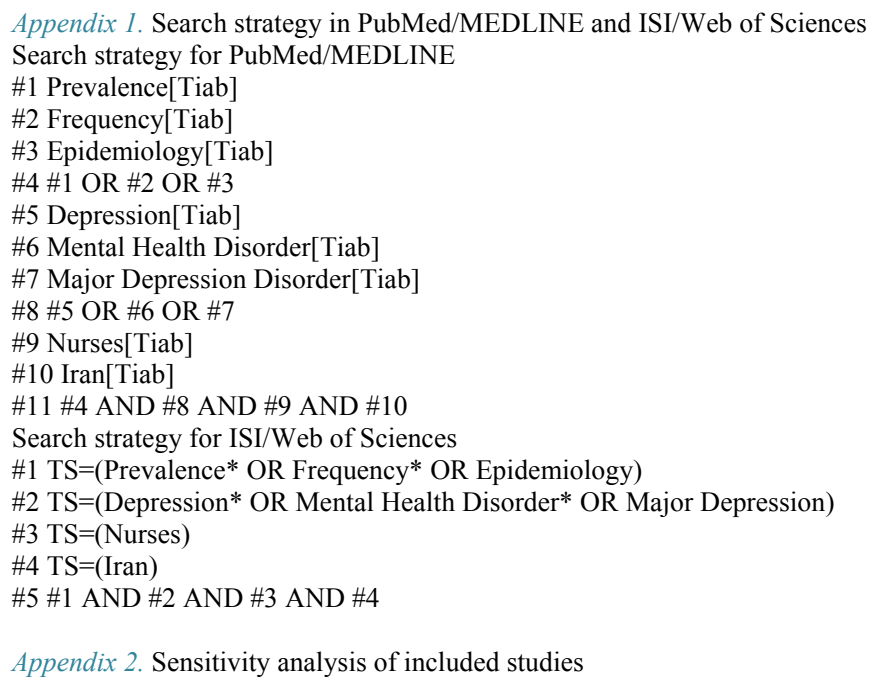

\section{Study}

Omitting Khajeh nasiri 2000

Omitting Khalil zadeh 2005

Omitting Habibzadeh 2005

Omitting Abedini 2007

Omitting Arefian 2009

Omitting Karami 2009

Omitting Dehghani 2009

Omitting Mirmohammadi 2009

Omitting Asadzandi 2011

Omitting Khamseh 2011

Omitting Baghiani moghadam 2012

Omitting Ghasemnegad 2012

Omitting Masoudi asl 2012

Omitting Hadavi 2012

Omitting Pournamdarian 2012

Omitting Mahmodi 2013

Omitting Nazemi 2013

Omitting Mami 2014

Omitting Kassani 2014

Omitting Taghva 2014

Omitting Taghinejad 2014

Omitting Tajvar 2015

Omitting Alipoor 2015

Omitting Yasemi 2015

Omitting Bahnam 2016

Omitting Khani 2016

Omitting Yazdanshenas 2016

Omitting Khodadadi 2016

Omitting Kashani 2017

Omitting Sagharjoghi Farahani 2017
Proportion $\quad 95 \%-\mathrm{Cl}$

$0.22[0.18 ; 0.27]$

$0.22[0.18 ; 0.27]$

$0.22[0.18 ; 0.26]$

$0.23[0.19 ; 0.27]$

$0.23[0.19 ; 0.27]$

$0.23[0.19 ; 0.27]$

$0.23[0.19 ; 0.27]$

$0.22[0.18 ; 0.26]$

$0.22[0.18 ; 0.27]$

$0.22[0.18 ; 0.27]$

$0.22[0.19 ; 0.27]$

$0.22[0.18 ; 0.26]$

$0.23[0.19 ; 0.28]$

$0.23[0.19 ; 0.27]$

$0.22[0.19 ; 0.27]$

$0.22[0.19 ; 0.27]$

$0.22[0.18 ; 0.26]$

$0.23[0.19 ; 0.28]$

$0.22[0.18 ; 0.27]$

$0.22[0.19 ; 0.27]$

$0.23[0.19 ; 0.28]$

$0.23[0.19 ; 0.27]$

$0.22[0.18 ; 0.27]$

$0.22[0.18 ; 0.26]$

$0.23[0.19 ; 0.28]$

$0.22[0.18 ; 0.27]$

$0.23[0.19 ; 0.28]$

$0.22[0.18 ; 0.26]$

$0.23[0.19 ; 0.28]$

$0.23[0.19 ; 0.27]$

$0.22[0.19 ; 0.27]$

Random effects model

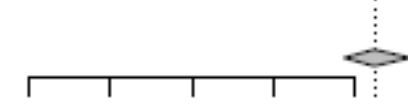


Appendix 3. Cumulative meta-analysis of included studies

Study

Adding Khajeh nasiri $2000(\mathrm{k}=1)$

Adding Khalil zadeh $2005(\mathrm{k}=2)$

Adding Habibzadeh 2005 ( $k=3$ )

Adding Abedini 2007 ( $\mathrm{k}=4$ )

Adding Arefian $2009(\mathrm{k}=5)$

Adding Karami 2009 ( $k=6)$

Adding Dehghani $2009(k=7)$

Adding Mirmohammadi 2009 ( $\mathrm{k}=8)$

Adding Asadzandi $2011(\mathrm{k}=9)$

Adding Khamseh 2011 ( $k=10)$

Adding Baghiani moghadam 2012 ( $\mathrm{k}=11$ )

Adding Ghasemnegad $2012(\mathrm{k}=12)$

Adding Masoudi asl $2012(\mathrm{k}=13)$

Adding Hadavi $2012(\mathrm{k}=14)$

Adding Pournamdarian $2012(\mathrm{k}=15)$

Adding Mahmodi 2013 ( $\mathrm{k}=16$ )

Adding Nazemi 2013 ( $\mathrm{k}=17)$

Adding Mami 2014 ( $\mathrm{k}=18$ )

Adding Kassani 2014 ( $(k=19)$

Adding Taghva $2014(\mathrm{k}=20)$

Adding Taghinejad $2014(\mathrm{k}=21)$

Adding Tajvar 2015 ( $\mathrm{k}=22)$

Adding Alipoor 2015 ( $\mathrm{k}=23$ )

Adding Yasemi $2015(\mathrm{k}=24)$

Adding Bahnam $2016(k=25)$

Adding Khani 2016 ( $\mathrm{k}=26)$

Adding Yazdanshenas 2016 ( $\mathrm{k}=27)$

Adding Khodadadi 2016 ( $\mathrm{k}=28$ )

Adding Kashani 2017 ( $k=29)$

Adding Sagharjoghi Farahani $2017(\mathrm{k}=30)$

Random effects model

\section{Proportion $\quad 95 \%-\mathrm{Cl}$}

$0.27[0.20 ; 0.36]$

$0.31[0.24 ; 0.40]$

$0.36[0.27 ; 0.48]$

$0.31[0.22 ; 0.43]$

$0.27[0.18 ; 0.40]$

$0.25[0.18 ; 0.37]$

$0.24[0.17 ; 0.34]$

$0.27[0.19 ; 0.38]$

$0.27[0.19 ; 0.37]$

$0.26[0.20 ; 0.35]$

$0.26[0.20 ; 0.34]$

$0.27[0.21 ; 0.34]$

$0.25[0.20 ; 0.32]$

$0.25[0.19 ; 0.32]$

$0.25[0.20 ; 0.31]$

$0.24[0.20 ; 0.31]$

$0.25[0.20 ; 0.31]$

$0.24[0.20 ; 0.30]$

$0.25[0.20 ; 0.30]$

$0.25[0.20 ; 0.30]$

$0.24[0.19 ; 0.29]$

$0.23[0.19 ; 0.28]$

$0.23[0.19 ; 0.28]$

$0.24[0.20 ; 0.29]$

$0.23[0.19 ; 0.28]$

$0.24[0.20 ; 0.28]$

$0.23[0.19 ; 0.28]$

$0.24[0.20 ; 0.28]$

$0.23[0.19 ; 0.27]$

$0.22[0.19 ; 0.27]$

$0.22[0.19 ; 0.27]$ 